\title{
Structures of fluorinated graphene and their signatures
}

\author{
H. Şahin, ${ }^{1}$ M. Topsakal, ${ }^{1}$ and S. Ciraci ${ }^{1,2, *}$ \\ ${ }^{1}$ UNAM-Institute of Materials Science and Nanotechnology, Bilkent University, 06800 Ankara, Turkey \\ ${ }^{2}$ Department of Physics, Bilkent University, 06800 Ankara, Turkey
}

(Received 4 February 2011; published 15 March 2011)

\begin{abstract}
Recent synthesis of fluorinated graphene introduced interesting stable derivatives of graphene. In particular, fluorographene (CF), namely, fully fluorinated chair conformation, is found to display crucial features, such as high mechanical strength, charged surfaces, local magnetic moments due to vacancy defects, and a wide band gap rapidly reducing with uniform strain. These properties, as well as structural parameters and electronic densities of states, are found to scale with fluorine coverage. However, most of the experimental data reported to date neither for $\mathrm{CF}$ nor for other $\mathrm{C}_{n} \mathrm{~F}$ structures complies with the results obtained from first-principles calculations. In this study, we attempt to clarify the sources of disagreements.
\end{abstract}

DOI: 10.1103/PhysRevB.83.115432 PACS number(s): 73.22.Pr, 61.48.Gh, 63.22.Rc, 71.20.-b

\section{INTRODUCTION}

Active research on graphene ${ }^{1}$ revealed not only numerous exceptional properties ${ }^{2-5}$ but also have prepared the grounds for the discovery of several graphene-based materials. Preparation of freestanding graphene sheets with nonuniform oxygen coverage have been achieved. ${ }^{6}$ More recently the synthesis of two-dimensional hydrocarbon in a honeycomb structure, so-called graphane ${ }^{7}(\mathrm{CH})$, showing diverse electronic, magnetic, and mechanical properties, ${ }^{8-12}$ is reported.

According to the Pauling scale, F has an electronegativity of 3.98, which is higher than that of $\mathrm{C}(2.55), \mathrm{H}(2.20)$, and $\mathrm{O}(3.44)$, and hence fluorination of graphene is expected to result in a material that may be even more interesting than both graphene oxide and $\mathrm{CH}$. Before the first synthesis of graphene, fluorinated graphite has been treated theoretically. ${ }^{13,14}$ Owing to promising properties revealed for $\mathrm{CH}$, fluorinated graphene structures are now attracting considerable interest $\mathrm{t}^{15-22}$ despite uncertainties in their chemical compositions and atomic structures. In an effort to identify the structures of fluorinated samples, previous theoretical models attempted to deduce the lowest-energy structures. ${ }^{13,15}$ In addition, band gaps of different structures calculated within density functional theory (DFT) are compared with the values revealed through specific measurements. ${ }^{17,18}$ However, the stability of proposed structures has not been questioned, and an underestimation of band gaps within DFT has not been studied. The Raman spectrum by itself has been limited in specifying $\mathrm{C}_{n} \mathrm{~F}$ structures. ${ }^{18}$

In this work, we first determined stable $\mathrm{C}_{n} \mathrm{~F}$ structures for $n \leqslant 4$. Then we revealed specific properties (such as internal structural parameters, elastic constants, the formation and binding energies, the energy band gap, and photoelectric threshold) for those stable structures as signatures to identify the derivatives probed experimentally. We placed an emphasis on fully fluorinated graphene or fluorographene (CF), in which $D$ and $G$ Raman peaks of bare graphene disappear after a long fluorination period. ${ }^{17,18}$ The present study reveals that the properties, such as structural parameters, binding energy, band gap, and phonon modes of various fluorinated structures, are strongly dependent on the binding structure of $F$ atoms and their composition. Some of these properties are found to roughly scale with $\mathrm{F}$ coverage. While the stable $\mathrm{C}_{2} \mathrm{~F}$ chair structure is metallic, $\mathrm{CF}$ is a nonmagnetic insulator with a band gap, $E_{g}$, being much larger than $3 \mathrm{eV}$, i.e., a value attributed experimentally to fully fluorinated graphene. In view of the calculated diffusion constant, Raman-active modes, and other properties, available experimental data suggest that domains (or grains) of various $\mathrm{C}_{n} \mathrm{~F}$ structures with extended and imperfect grain boundaries can coexist after the fluorination process. Hence the measured properties are averaged from diverse perfect and imperfect regions.

\section{COMPUTATIONAL METHODOLOGY}

Our predictions are obtained from first-principles planewave calculations ${ }^{23}$ within DFT, which is demonstrated to yield rather accurate results for carbon-based materials. Calculations are performed using the spin-polarized local-density approximation (LDA) ${ }^{24}$ and projector augmented wave (PAW) potentials. ${ }^{25}$ The kinetic energy cutoff $\hbar^{2}|\mathbf{k}+\mathbf{G}|^{2} / 2 m$ for a plane-wave basis set is taken as $500 \mathrm{eV}$. In the self-consistent potential and total energy calculations of fluorographene a set of $(25 \times 25 \times 1) \mathbf{k}$-point samplings is used for Brillouin zone (BZ) integration. The convergence criterion of self-consistent calculations for ionic relaxations is $10^{-5} \mathrm{eV}$ between two consecutive steps. By using the conjugate gradient method, all atomic positions and unit cells are optimized until the atomic forces are less than $0.03 \mathrm{eV} / \AA$. Pressures on the lattice unit cell are decreased to values less than $0.5 \mathrm{kbar}$. The energy band gap, which is usually underestimated in DFT, is corrected by frequency-dependent $G W_{0}$ calculations. ${ }^{26}$ In $G W_{0}$ corrections screened Coulomb potential, $W$, is kept fixed to an initial DFT value $W_{0}$ and the Green's function, $G$, is iterated four times. Various tests are performed regarding vacuum spacing, kinetic energy cutoff energy, number of bands, $\mathbf{k}$ points, and grid points. Finally, the band gap of $\mathrm{CF}$ is found $7.49 \mathrm{eV}$ after $G W_{0}$ correction, which is carried out by using $(12 \times 12 \times 1)$ k points in BZ, a $15-\AA$ vacuum spacing, a default cutoff potential for $G W_{0}, 192$ bands, and 64 grid points. Phonon frequencies and phonon eigenvectors are calculated using the density functional perturbation theory (DFPT). ${ }^{27}$

\section{STRUCTURES OF FLUORINATED GRAPHENE}

Each carbon atom of graphene can bind only one F atom, and through coverage (or decoration) of one or two sides of 
graphene, one can achieve diverse $\mathrm{C}_{n} \mathrm{~F}$ structures. Uniform $\mathrm{F}$ coverage is specified by $\Theta=1 / n$ (namely, one $\mathrm{F}$ adatom per $n \mathrm{C}$ atoms), whereby $\Theta=0.5$ corresponds to half fluorination and $\Theta=1$ is fluorographene CF. The adsorption of a single $\mathrm{F}$ atom to graphene is a precursor for fluorination. When placed at diverse sites of a $(4 \times 4)$ supercell of graphene, a simple F atom moves to the top site of a carbon atom and remains adsorbed there. The resulting structure is nonmagnetic and its binding energy is $E_{b}=2.71 \mathrm{eV}$ in equilibrium, which is a rather strong binding unlike many other adatoms adsorbed to graphene. An energy barrier, $Q_{B}=\sim 0.45 \mathrm{eV}$, occurs along its minimum energy migration path. Our calculations, related with the minimum energy path of a single $\mathrm{F}$ atom, follow hexagons of the underlying graphene. Namely, the $F$ atom migrates from the highest binding energy site, i.e., the top site (on top of the carbon atom) to the next top site through a bridge site (the bridge position between two adjacent carbon atoms of graphene). The corresponding diffusion constant for a single F atom, $D=v a e^{-Q_{B} / k_{B} T}$, is calculated in terms of the lattice constant, $a=2.55 \AA$, and characteristic jump frequency $v \approx$ $39 \mathrm{THz}$. Experiments present evidence that energy barriers on the order of $0.5 \mathrm{eV}$ would make the adatoms mobile. ${ }^{18,28}$ Moreover, this energy barrier is further lowered even it is collapsed in the presence of a second $\mathrm{F}$ atom at close proximity.
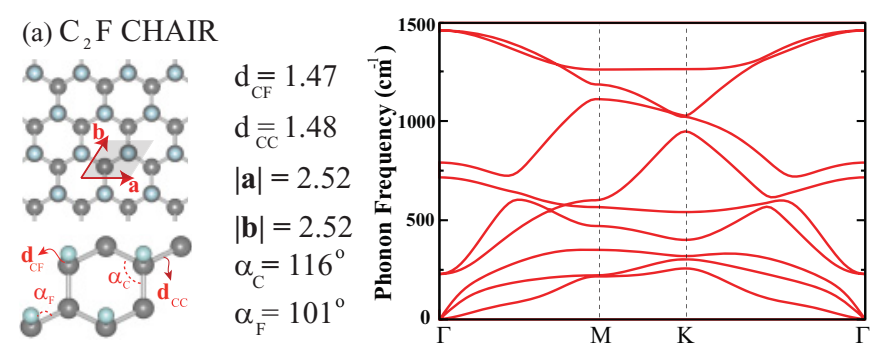

(b) $\mathrm{C}_{2} \mathrm{~F}$ BOAT
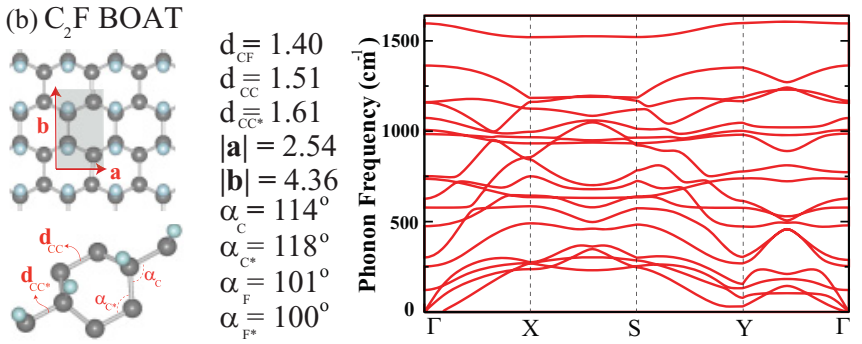

(c) $\mathrm{C}_{4} \mathrm{~F}$
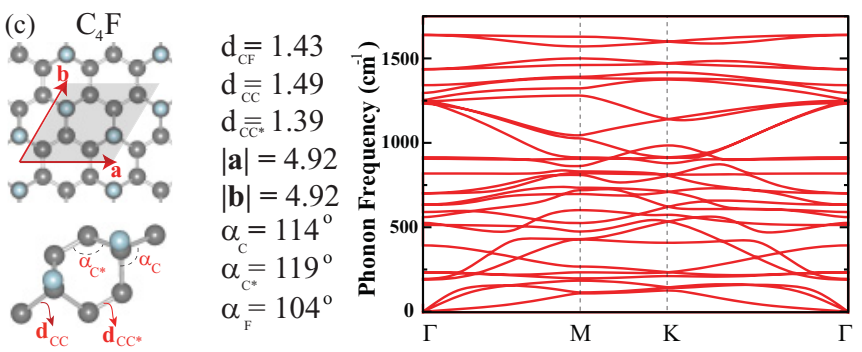

FIG. 1. (Color online) Atomic structure and calculated phonon bands (i.e., phonon frequencies vs wave vector, k) of various optimized $\mathrm{C}_{n} \mathrm{~F}$ structures calculated along the symmetry directions of BZ. Carbon and fluorine atoms are indicated by black (dark) and blue (light) balls, respectively. (a) $\mathrm{C}_{2} \mathrm{~F}$ chair structure. (b) $\mathrm{C}_{2} \mathrm{~F}$ boat structure. (c) $\mathrm{C}_{4} \mathrm{~F}$ structure. Units are $\AA$ for structural parameters and $\mathrm{cm}^{-1}$ for frequencies.
Consequently, this situation, together with the tendency toward clustering, favors that $\mathrm{C}_{n} \mathrm{~F}$ grains (or domains) of different $n$ on graphene can form during the course of fluorination. We note that the energy barrier for the diffusion of a single carbon adatom adsorbed on the bridge sites of graphene was calculated to be in a similar energy range. Carbon adatoms on graphene were found to be rather mobile. That energy barrier for a single $\mathrm{C}$ adatom was found to decrease, and even to collapse at a close proximity to a second adatom. ${ }^{29}$

In earlier theoretical studies, ${ }^{13,15,17}$ the total energies and/or binding energies were taken as the criteria for whether a given $\mathrm{C}_{n} \mathrm{~F}$ structure exists. Even if a $\mathrm{C}_{n} \mathrm{~F}$ structure seems to be in a minimum on the Born-Oppenheimer surface, its stability is meticulously examined by calculating frequencies of all phonon modes in BZ. Here we calculated phonon dispersions of most of the optimized $\mathrm{C}_{n} \mathrm{~F}$ structures. We found that the $\mathrm{C}_{4} \mathrm{~F}$, the $\mathrm{C}_{2} \mathrm{~F}$ boat, the $\mathrm{C}_{2} \mathrm{~F}$ chair (see Fig. 1), and the $\mathrm{CF}$ chair (see Fig. 2) structures have positive frequencies throughout the $\mathrm{BZ}$, indicating their stability.

Some of phonon branches of $\mathrm{C}_{n} \mathrm{~F}$ structures (for example, the $\mathrm{CF}$ boat) have imaginary frequencies and hence are unstable, in spite of the fact that their structures can be optimized. The possibility that these unstable structures can occur at finite and small sizes is, however, not excluded. For stable structures, the gap between optical and acoustical branches is collapsed, since the optical branches

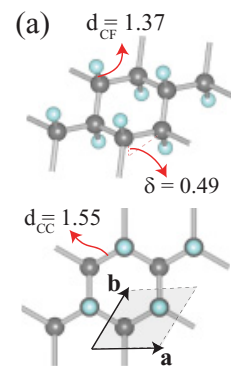

(c)

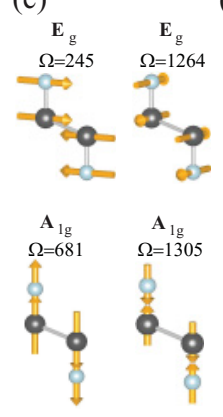

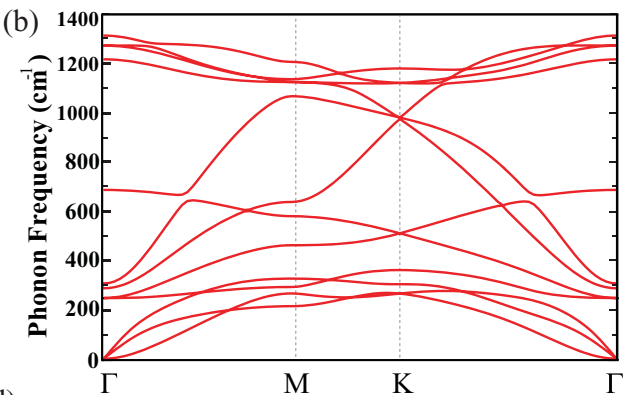

(d)

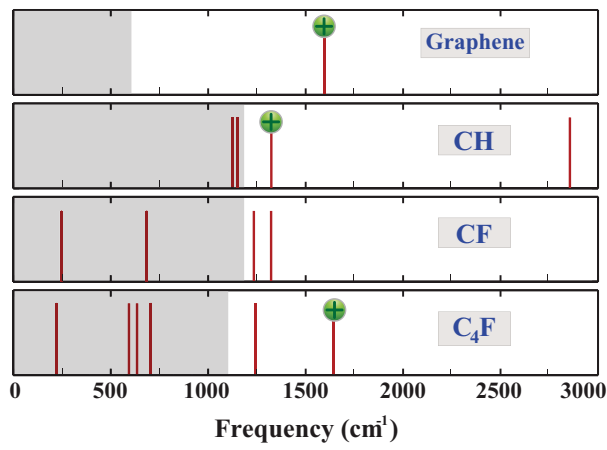

FIG. 2. (Color online) (a) Atomic structure of fluorographene CF. $a$ and $b$ are the lattice vectors $(|a|=|b|)$ of a hexagonal structure; $d_{\mathrm{CC}}\left(d_{\mathrm{CF}}\right)$ is the $\mathrm{C}-\mathrm{C}(\mathrm{C}-\mathrm{F})$ bond distance; $\delta$ is the buckling. (b) Phonon frequencies vs wave vector $\mathbf{k}$ of optimized CF calculated along symmetry directions in BZ. (c) Symmetries, frequencies, and descriptions of Raman-active modes of $\mathrm{CF}$. (d) Calculated Raman-active modes of graphene, $\mathrm{CH}, \mathrm{CF}$, and $\mathrm{C}_{4} \mathrm{~F}$ are indicated on the frequency axis. Those modes indicated by " + " are observed experimentally. There is no experimental Raman data in the shaded regions. Units are $\AA$ for structural parameters and $\mathrm{cm}^{-1}$ for frequencies. 
TABLE I. Comparison of the calculated properties of four stable, fluorinated graphene structures (namely, $\mathrm{CF}$, the $\mathrm{C}_{2} \mathrm{~F}$ chair, the $\mathrm{C}_{2} \mathrm{~F}$ boat, and $\mathrm{C}_{4} \mathrm{~F}$ ) with those of graphene and $\mathrm{CH}$. Lattice constant, $a=b\left(a \neq b\right.$ for rectangular lattice); $\mathrm{C}-\mathrm{C}$ bond distance, $d_{\mathrm{CC}}$ (second entries with the slash differ from the previous one); $\mathrm{C}-\mathrm{X}$ bond distance $[\mathrm{X}$ indicating $\mathrm{H}(\mathrm{F})$ atom for $\mathrm{CH}(\mathrm{CF})], d_{\mathrm{CX}}$; the buckling, $\delta$; angle between adjacent $\mathrm{C}-\mathrm{C}$ bonds, $\alpha_{\mathrm{C}}$; angle between adjacent $\mathrm{C}-\mathrm{X}$ and $\mathrm{C}-\mathrm{C}$ bonds, $\alpha_{\mathrm{X}}$; total energy per cell comprising eight carbon atoms $E_{T}$; formation energy per $\mathrm{X}$ atom relative to graphene, $E_{f}$; binding energy per $\mathrm{X}$ atom relative to graphene, $E_{b}$ (the value in parentheses, $E_{b^{\prime}}$, excludes the $\mathrm{X}-\mathrm{X}$ coupling); desorption energy, $E_{d}$ (see the text for formal definitions); energy band gap calculated by LDA, $E_{g}^{\mathrm{LDA}}$; energy band gap corrected by $G W_{0}, E_{g}^{G W_{0}}$; photoelectric threshold, $\Phi$; in-plane stiffness, $C$; Poisson ratio, $v$. All materials are treated in a hexagonal lattice, except for the $\mathrm{C}_{2} \mathrm{~F}$ boat, which has a rectangular lattice.

\begin{tabular}{lccccccccccccccc}
\hline \hline Material & $\begin{array}{c}a(b) \\
(\AA)\end{array}$ & $\begin{array}{c}d_{\mathrm{CC}} \\
(\AA)\end{array}$ & $\begin{array}{c}d_{\mathrm{CX}} \\
(\AA)\end{array}$ & $\begin{array}{c}\delta \\
(\AA)\end{array}$ & $\begin{array}{c}\alpha_{\mathrm{C}} \\
(\mathrm{deg})\end{array}$ & $\begin{array}{c}\alpha_{\mathrm{X}} \\
(\mathrm{deg})\end{array}$ & $\begin{array}{c}E_{g}^{\mathrm{LDA}} \\
(\mathrm{eV})\end{array}$ & $\begin{array}{c}E_{g}^{G W_{0}} \\
(\mathrm{eV})\end{array}$ & $\begin{array}{c}E_{T} \\
(\mathrm{eV})\end{array}$ & $\begin{array}{c}E_{f} \\
(\mathrm{eV})\end{array}$ & $\begin{array}{c}E_{b}\left(E_{b^{\prime}}\right) \\
(\mathrm{eV})\end{array}$ & $\begin{array}{c}E_{d} \\
(\mathrm{eV})\end{array}$ & $\begin{array}{c}\Phi \\
(\mathrm{eV})\end{array}$ & $\begin{array}{c}C \\
\left(\mathrm{~J} / \mathrm{m}^{2}\right)\end{array}$ & $\begin{array}{c}v \\
v\end{array}$ \\
\hline Graphene (Ref. 30) & 2.46 & 1.42 & - & 0.00 & 120 & - & 0.00 & 0.00 & -80.73 & - & - & - & 4.77 & 335 & 0.16 \\
$\mathrm{CH}$ (Ref. 10) & 2.51 & 1.52 & 1.12 & 0.45 & 112 & 107 & 3.42 & 5.97 & -110.56 & 0.39 & $2.8(2.5)$ & 4.8 & 4.97 & 243 & 0.07 \\
$\mathrm{CF}$ & 2.55 & 1.55 & 1.37 & 0.49 & 111 & 108 & 2.96 & 7.49 & -113.32 & 2.04 & $3.6(2.9)$ & 5.3 & 7.94 & 250 & 0.14 \\
$\mathrm{C}_{2} \mathrm{~F}$ chair & 2.52 & 1.48 & 1.47 & 0.29 & 116 & 101 & Metal Metal & -89.22 & 0.09 & $1.7(0.9)$ & 1.2 & $8.6 / 5.6$ & 280 & 0.18 \\
$\mathrm{C}_{2} \mathrm{~F}$ boat & $2.54(4.36)$ & $1.51 / 1.61$ & 1.40 & 0.42 & $114 / 118$ & $100 / 101$ & 1.57 & 5.68 & -92.48 & 0.91 & $2.5(1.6)$ & 2.4 & $7.9 / 5.1$ & $286(268)$ & 0.05 \\
$\mathrm{C}_{4} \mathrm{~F}$ & 4.92 & $1.49 / 1.39$ & 1.43 & 0.34 & $114 / 119$ & 104 & 2.93 & 5.99 & -87.68 & 1.44 & $3.0(2.7)$ & 3.5 & $8.1 / 5.6$ & 298 & 0.12 \\
\hline \hline
\end{tabular}

associated with the modes of $\mathrm{C}-\mathrm{F}$ bonds occur at lower frequencies. This situation is in contrast with the phonon spectrum of graphane, ${ }^{10}$ where optical modes related with C-H bonds appear above the acoustical branches at $\sim 2900$ $\mathrm{cm}^{-1}$.

The formation energy of fluorination is defined as $E_{f}=$ $\left(n_{\mathrm{F} 2} E_{T, \mathrm{~F}_{2}}+E_{T, \mathrm{Gr}}-E_{T, \mathrm{C}_{n} F}\right) / n_{\mathrm{F}}$ in terms of the total groundstate energies of optimized structures of graphene and fluorinated graphenes at different compositions, respectively, $E_{T, \mathrm{Gr}}, E_{T, \mathrm{C}_{n} \mathrm{~F}}$, and the total ground-state energy of a single carbon atom, $E_{T, \mathrm{C}}$, of a $\mathrm{F}_{2}$ molecule and a $\mathrm{F}$ atom, $E_{T, \mathrm{~F}_{2}}$ and $E_{T, \mathrm{~F}}$. Similarly, the binding energy of the $\mathrm{F}$ atom relative to graphene including $\mathrm{F}-\mathrm{F}$ coupling is $E_{b}=\left(E_{T, \mathrm{Gr}}+n_{\mathrm{F}} E_{T, \mathrm{~F}}-E_{T, \mathrm{C}_{n} \mathrm{~F}}\right) / n_{\mathrm{F}}$ and without F-F coupling $E_{b^{\prime}}=\left(E_{T, \mathrm{Gr}}+E_{T, n_{\mathrm{F}} \mathrm{F}}-E_{T, \mathrm{C}_{n} \mathrm{~F}}\right) / n_{\mathrm{F}}$. Here $E_{T, n_{\mathrm{F}} \mathrm{F}}$ is the total energy of suspended single or double layers of $\mathrm{F}$ occupying the same positions as in $\mathrm{C}_{n} \mathrm{~F}$. The desorption energy, $E_{d}$ is the energy required to remove one single $\mathrm{F}$ atom from the surface of $\mathrm{C}_{n} \mathrm{~F} . n_{\mathrm{F} 2}$ and $n_{\mathrm{F}}$ are numbers of $\mathrm{F}_{2}$ molecules and $\mathrm{F}$ atoms, respectively. The total energies are calculated in periodically repeating supercells comprising eight carbon atoms and keeping all the parameters of calculations described above using spin-polarized as well as spin-unpolarized LDA. The lowest (magnetic or nonmagnetic) total energy is used as the ground-state total energy.

Fluorographene $(\mathrm{CF})$, where $\mathrm{F}$ atoms are bound to each $\mathrm{C}$ atom of graphene alternatingly from top and bottom sides, is energetically the most favorable structure. Upon full fluorination, the planar honeycomb structure of $\mathrm{C}$ atoms becomes buckled (puckered) and the C-C bond length increases by $\sim 10 \%$. At the end, while planar $s p^{2}$ bonding of graphene is dehybridized, the buckled configuration is maintained by $s p^{3}$-like rehybridization. In Table I, the calculated lattice constants, internal structural parameters, relevant binding energies, and energy band gaps of stable $\mathrm{C}_{n} \mathrm{~F}$ structures are compared with those of bare graphene and $\mathrm{CH} .{ }^{10}$ Notably, internal parameters (such as $\delta, \mathrm{C}-\mathrm{C}$ bond length) as well as lattice constants of various $\mathrm{C}_{n} \mathrm{~F}$ structures vary with $\mathrm{F}$ coverage, $\Theta$. CF has the highest values for $E_{f}, E_{b}, E_{b^{\prime}}$, and $E_{d}$ given in Table I; those of $\mathrm{C}_{4} \mathrm{~F}$ are second highest among stable $\mathrm{C}_{n} \mathrm{~F}$ structures.
Since the Raman spectrum can convey information for a particular structure and hence can set its signature, the calculated Raman-active modes of stable $\mathrm{C}_{4} \mathrm{~F}$ and $\mathrm{CF}$ structures, together with those of graphene and $\mathrm{CH}$, are also indicated in Figs. 2(c) and 2(d). It is known that the only characteristic Raman active mode of graphene at $1594 \mathrm{~cm}^{-1}$ is observed so far. $^{31}$ Similarly, for $\mathrm{CH}$ the mode at $\sim 1342$ $\mathrm{cm}^{-1}$ is observed. ${ }^{7}$ One of two Raman-active modes of $\mathrm{C}_{4} \mathrm{~F}$ at $1645 \mathrm{~cm}^{-1}$ seems to be observed. ${ }^{17}$ In compliance with the theory, ${ }^{32}$ phonon branches of all these observed modes exhibit a kink structure. However, none of the Raman active modes of CF revealed in Fig. 2 has been observed yet. Raman spectroscopy in the low-frequency range may be useful in identifying experimental structures.

\section{ELECTRONIC STRUCTURES}

Energy bands, which are calculated for the optimized $\mathrm{C}_{4} \mathrm{~F}$, the $\mathrm{C}_{2} \mathrm{~F}$ boat, the $\mathrm{C}_{2} \mathrm{~F}$ chair, and the $\mathrm{CF}$ chair structures are presented in Figs. 3 and 4, respectively. The orbital projected densities of states (PDOS), together with the total densities of states of these optimized structures, are also presented. An analysis of the electronic structure can also provide data to reveal the observed structure of the fluorinated graphene. As seen in Table I, stable $\mathrm{C}_{n} \mathrm{~F}$ structures have LDA band gaps ranging from 0 to $2.96 \mathrm{eV}$. Surprisingly, the $\mathrm{C}_{2} \mathrm{~F}$ chair structure is found to be a metal owing to the odd number of valence electrons in the primitive unit cell. Even if various measurements on the band gap of fluorinated graphene lie in the energy range from $68 \mathrm{meV}$ (Ref. 16) to $3 \mathrm{eV},{ }^{18}$ these calculated band gaps are underestimated by LDA. Incidentally, the band gaps change significantly after they are corrected by various self-energy methods. In fact, the correction using the $G W_{0}$ self-energy method predicts a rather wide band gap of $7.49 \mathrm{eV}$ for $\mathrm{CF}$. The corrected band gaps for the $\mathrm{C}_{2} \mathrm{~F}$ boat structure and $\mathrm{C}_{4} \mathrm{~F}$ are 5.68 and $5.99 \mathrm{eV}$, respectively. It should be noted that the $G W_{0}$ self-energy method has been successful in predicting the band gaps of three-dimensional (3D) semiconductors. ${ }^{33}$

While predicting a much larger band gap for $\mathrm{CF}$, the measured band gap of $\sim 3 \mathrm{eV}$ reported by Nair et al. ${ }^{18}$ marks the serious discrepancy between theory and experiment. The 

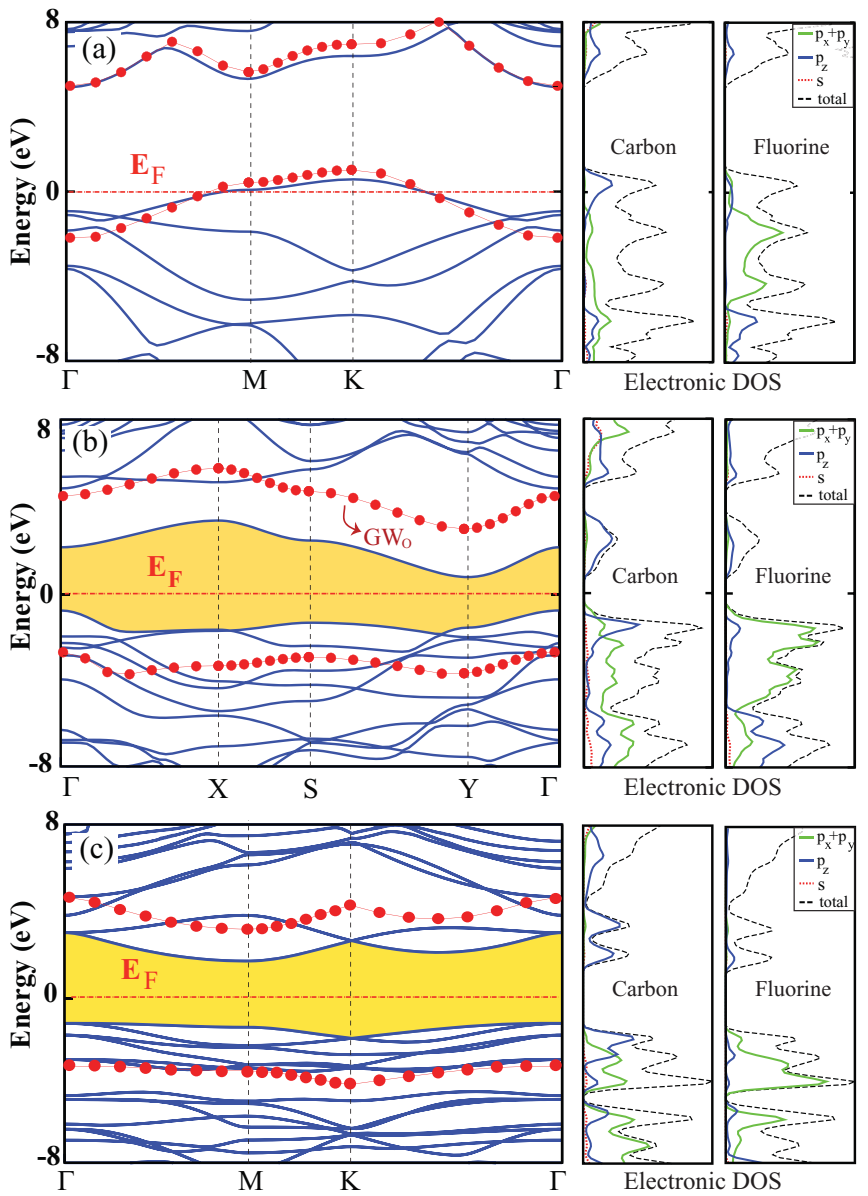

FIG. 3. (Color online) Energy band structures of various stable $\mathrm{C}_{n} \mathrm{~F}$ structures, together with the orbital PDOS and the total densities of states (DOS). The LDA band gaps are shaded and the zero of energy is set to the Fermi level $E_{F}$. The total DOS is scaled to $45 \%$. Valenceand conduction-band edges after $G W_{0}$ correction are indicated by filled (red) circles. (a) $\mathrm{C}_{2} \mathrm{~F}$ chair structure. (b) $\mathrm{C}_{2} \mathrm{~F}$ boat structure. (c) $\mathrm{C}_{4} \mathrm{~F}$ structure.

character of the band structure of $\mathrm{CF}$ is revealed from the analysis of PDOS as well as charge densities of specific bands in Fig. 4(b). The conduction-band edge consists of the antibonding combination of $p_{z}$ orbitals of $\mathrm{F}$ and $\mathrm{C}$ atoms. The $p_{z}$ orbitals of $\mathrm{C}$ atoms by themselves, are combined to form $\pi$ bands. The bands at the edge of the valence band are derived from the combination of C- $\left(p_{x}+p_{y}\right)$ and F- $\left(p_{x}+p_{y}\right)$ orbitals. The total contribution of the $\mathrm{C}$ orbitals to the valence band can be viewed as the contribution of four tetrahedrally coordinated $s p^{3}$-like hybrid orbitals of the $s$ and $p$ orbitals of the $\mathrm{C}$ atoms. However, the deviation from tetrahedral coordination increases when $n$ increases or the single side is fluorinated. As a matter of fact, the total DOS presented in Figs. 3 and 4 marks crucial differences. In this respect, spectroscopy data is expected to yield significant information regarding the observed structures of fluorinated graphenes.

The contour plots of the total charge density, $\rho_{T}$, in the F-C-C-F plane suggests the formation of strong covalent C-C bonds from the bonding combination of two C-s $p^{3}$ hybrid orbitals. The difference charge density, $\Delta \rho$ (which is obtained by subtracting the charges of free $\mathrm{C}$ and free $\mathrm{F}$ atoms situated (a)

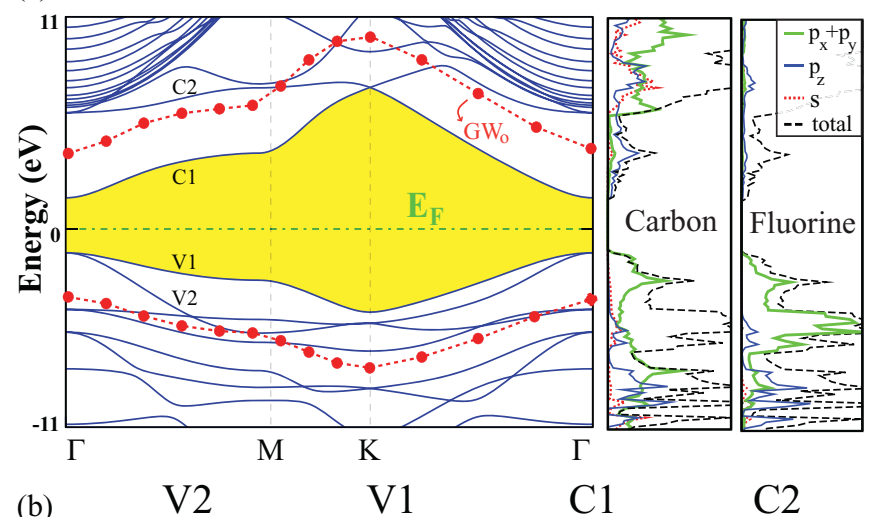

(b)
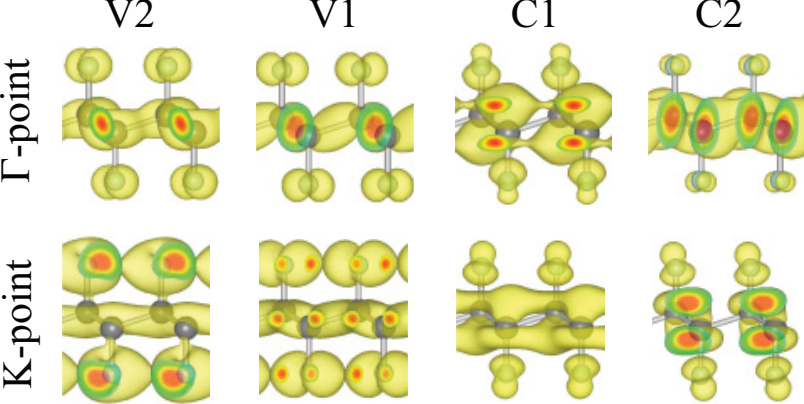

(c)
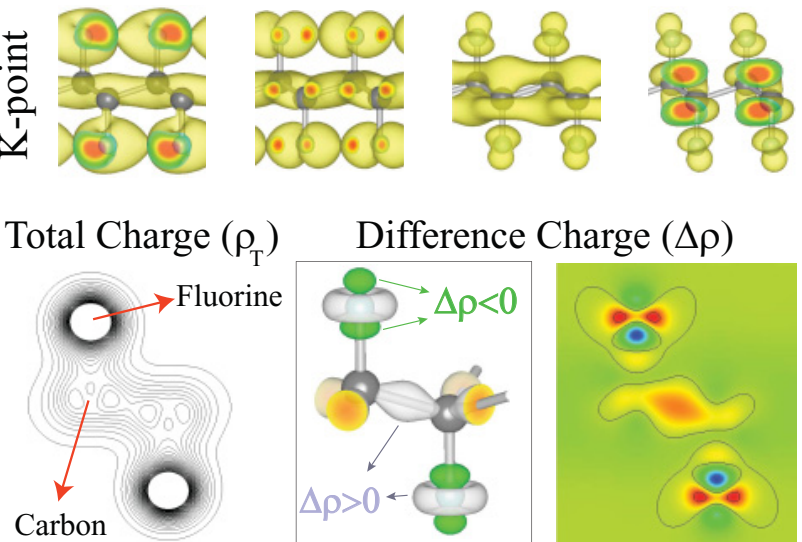

Difference Charge $(\Delta \rho)$
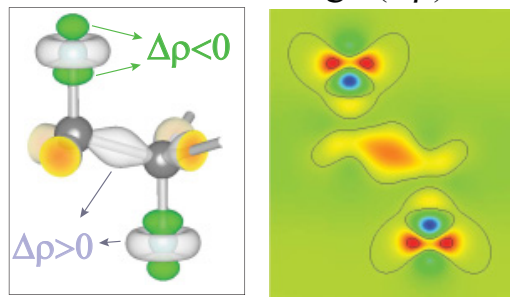

FIG. 4. (Color online) (a) Energy-band structure of CF, together with the orbital PDOS and total DOS. The LDA band gap is shaded and the zero of energy is set to the Fermi level, $E_{F}$. Valence- and conduction-band edges after $G W_{0}$ correction are indicated by filled (red) circles. (b) Isosurfaces of charge DOS corresponds to first (V1), second (V2) valence and first (C1) and second (C2) conduction bands at the $\Gamma$ and $K$ points. (c) Contour plots of the total charge density $\rho_{T}$ and difference charge density $\Delta \rho$ in the plane passing through F-C-C-F atoms. Contour spacings are $0.03 e / \AA^{3}$.

at their respective positions in $\mathrm{CF}$ ), indicates charge transfer to the middle of the $\mathrm{C}-\mathrm{C}$ bond and to $\mathrm{F}$ atom, revealing the bond charge between $\mathrm{C}$ atoms and the ionic character of the $\mathrm{C}-\mathrm{F}$ bond. However, the value of the charge transfer is not unique, but diversifies among different methods of analysis. ${ }^{34}$ Nevertheless, the direction of the calculated charge transfer is in compliance with the Pauling ionicity scale and is corroborated by calculated Born effective charges, which have in-plane $(\|)$ and out-of-plane $(\perp)$ components on $\mathrm{C}$ atoms, $Z_{\mathrm{C}, \|}^{*}=0.30, Z_{\mathrm{C}, \perp}^{*}=0.35$ and on $\mathrm{F}$ atoms $Z_{\mathrm{F}, \|}^{*}=-0.30$, $Z_{\mathrm{F}, \perp}^{*}=-0.35$.

Finally, we note that a perfect $\mathrm{CF}$ is a nonmagnetic insulator. However, a single isolated $\mathrm{F}$ vacancy attains a net magnetic moment of 1 Bohr magneton $\left(\mu_{B}\right)$ and localized defect states in the band gap. Creation of an unpaired $\pi$ electron upon $F$ vacancy is the source of a magnetic moment. However, the 
exchange interaction between two $F$ vacancies calculated in a $(7 \times 7 \times 1)$ supercell is found to be nonmagnetic for the first-nearest-neighbor distances due to spin pairings. Similar to graphane, ${ }^{10,11}$ it is also possible to attain large magnetic moments on F-vacant domains in CF structures.

\section{ELASTIC PROPERTIES OF CF}

Having analyzed the stability of various $\mathrm{C}_{n} \mathrm{~F}$ structures with $n=1,2$, and 4, we next investigate their mechanical properties. The elastic properties of this structure can be conveniently characterized by its Young's modulus and Poisson's ratio. However, the in-plane stiffness $C$ is known to be a better measure of the strength of single-layer honeycomb structures, since the thickness of the layer $h$ cannot be defined unambiguously. Defining $A_{0}$ as the equilibrium area of a $\mathrm{C}_{n} \mathrm{~F}$ structure, the in-plane stiffness is obtained as $C=\left(\delta E_{s}^{2} / \delta \epsilon^{2}\right) / A_{0}$, in terms of strain energy $E_{s}$ and uniaxial strain $\epsilon{ }^{12}$ The values of in-plane stiffness $C$, and Poisson's ratio $v$, calculated for stable $\mathrm{C}_{n} \mathrm{~F}$ structures, are given in Table I together with the values calculated for graphene and graphane. For example, the calculated values of CF are $C=250 \mathrm{~J} / \mathrm{m}^{2}$ and $v=0.14$. It is noted that $C$ increases with $n$. For $\mathrm{CF}$ (i.e., $n=1$ ), the in-plane stiffness is close to that calculated for $\mathrm{CH}$. It appears that the interaction between $\mathrm{C}-\mathrm{F}$ bonds in $\mathrm{CF}$ (or the interaction between $\mathrm{C}-\mathrm{H}$ bonds in $\mathrm{CH}$ ) does not have a significant contribution to the in-plane stiffness. The main effect occurs through dehybridization of $s p^{2}$ bonds of graphene through the formation $\mathrm{C}-\mathrm{F}$ bonds (or $\mathrm{C}-\mathrm{H}$ bonds).

A value of the Young's modulus of $\sim 0.77 \mathrm{TPa}$ can be calculated by estimating the thickness of CF as $h=3.84 \AA$, namely the sum of the thickness of graphene $(3.35 \AA)$ and buckling, $\delta(0.49 \AA)$. This value is smaller but comparable with the value proposed for graphene, i.e., $\sim 1 \mathrm{TPa}$. Here the contribution of C-F bonds to the thickness of $\mathrm{CF}$ is neglected, since the interaction between $\mathrm{C}-\mathrm{F}$ bonds has only negligible effects on the strength of CF.

In Fig. 5 the variation of strain energy $E_{s}$ and its derivative, $\delta E_{s} / \delta \epsilon$, with strain $\epsilon$ are presented in both elastic and plastic regions. Two critical strain values, $\epsilon_{c_{1}}$ and $\epsilon_{c_{2}}$, are deduced. The first one, $\epsilon_{c_{1}}$, is the point where the derivative curve attains its maximum value. This means that the structure can
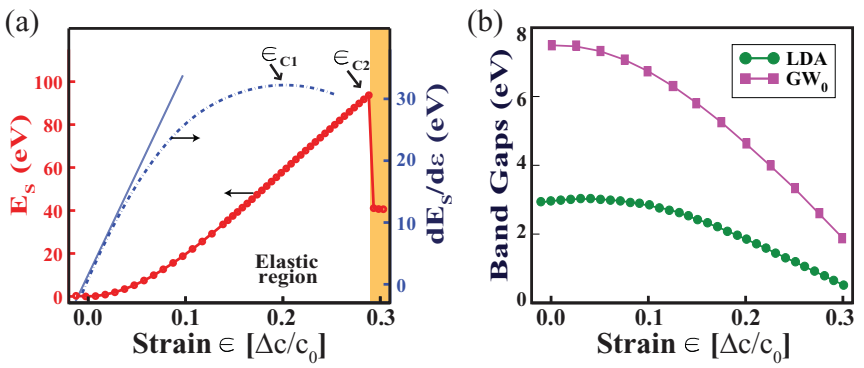

FIG. 5. (Color online) (a) Variation of strain energy and its first derivative with respect to the uniform strain $\epsilon$. Orange (gray) shaded region indicates the plastic range. Two critical strains in the elastic range are labeled as $\epsilon_{c 1}$ and $\epsilon_{c 2}$. (b) Variation of the band gaps with $\epsilon$. LDA and $G W_{0}$ calculations are carried out using a $5 \times 5$ supercell having a lattice parameter of $c_{0}=5 a$, and $\Delta c$ is its stretching be expanded under a smaller tension for higher values of strain. This point also corresponds to phonon instability ${ }^{12}$ where the longitudinal acoustic modes start to become imaginary for $\epsilon>\epsilon_{c_{1}}$. The second critical point, $\epsilon_{c_{2}}(\simeq 0.29)$, corresponds to the yielding point. Until this point the honeycomblike structure is preserved, but beyond it the plastic deformation sets in. We note that for $\epsilon_{c_{1}}<\epsilon<\epsilon_{c_{2}}$ the system is actually in a metastable state, where the plastic deformation is delayed. Under long-wavelength perturbations, vacancy defects and high ambient temperatures, $\epsilon_{c_{2}}$ approaches to $\epsilon_{c_{1}}$. In fact, our further molecular dynamics simulations show that $\epsilon_{c_{2}} \rightarrow$ 0.17 at $300 \mathrm{~K}$ and to 0.16 at $600 \mathrm{~K}$. In the presence of a periodically repeating $\mathrm{F}$ vacancy and $\mathrm{C}+\mathrm{F}$ divacancy, the value of $\epsilon_{c_{2}}$ is also lowered to 0.21 and 0.14 , respectively. Apart from phonon instability occurring at high $\epsilon$, the band gap is strongly affected under uniform expansion. In Fig. 5(b) we show the variation of LDA and $G W_{0}$-corrected band gaps under uniform expansion. The LDA gap slightly increases until $\epsilon=0.05$ and then decreases steadily with increasing $\epsilon$. The $G W_{0}$-corrected band gap essentially decreases with increasing strain. For example, its value decreases by $38 \%$ for $\epsilon=0.20$.

\section{CONCLUSIONS}

The present analysis of fluorinated graphenes shows that different $\mathrm{C}_{n} \mathrm{~F}$ structures can form at different levels of $\mathrm{F}$ coverage. Calculated properties of these structures, such as lattice parameter, $d_{\mathrm{CC}}$ distance, band gap, DOS, work function, in-plane stiffness $C$, Poisson's ratio, and surface charge, are shown to depend on $n$ or coverage $\Theta$. Relevant data reported in various experiments do not appear to agree with the properties calculated for any one of the stable $\mathrm{C}_{n} \mathrm{~F}$ structures. This finding leads us to conclude that domains of various $\mathrm{C}_{n} \mathrm{~F}$ structures can form in the course of the fluorination of graphene. Therefore, the experimental data may reflect a weighted average of diverse $\mathrm{C}_{n} \mathrm{~F}$ structures, together with extended defects in grain boundaries. In this respect, imaging of fluorinated graphene surfaces by scanning tunneling and atomic force microscopy, as well as X-ray photoemission spectroscopy, is expected to shed light on the puzzling inconsistency between theory and experiment.

Finally, our results show a wide range of interesting features of $\mathrm{C}_{n} \mathrm{~F}$ structures. For example, a perfect $\mathrm{CF}$ structure, as described in Fig. 2, is a stiff, nonmagnetic, wide-band-gap nanomaterial having a substantial surface charge, but attains a significant local magnetic moment through F-vacancy defects. Moreover, unlike graphane, half-fluorinated graphene with only one side fluorinated is found to be stable, which can be further functionalized by the adsorption of adatoms to the other side. For example, hydrogen atoms adsorbed to the other side attain a positive charge and hence a permanent transversal electric field, which can be utilized to engineer electronic properties.

\section{ACKNOWLEDGMENTS}

This work is supported by TUBITAK through Grant No. 108T234. Part of the computational resources has been provided by UYBHM at ITU through Grant No. 2-024-2007. 
We thank the DEISA Consortium (www.deisa.eu), funded through the EU FP7 project RI-222919, for support within the DEISA Extreme Computing Initiative. S.C. acknowledges the partial support of TUBA, Academy of Science of Turkey. The authors would also like to acknowledge the valuable suggestions made by D. Alfe. *ciraci@fen.bilkent.edu.tr

${ }^{1}$ K. S. Novoselov, A. K. Geim, S. V. Morozov, D. Jiang, Y. Zhang, S. V. Dubonos, I. V. Grigorieva, and A. A. Firsov, Science 306, 666 (2004).

${ }^{2}$ A. K. Geim and K. S. Novoselov, Nat. Mater. 6, 183 (2007).

${ }^{3}$ C. Berger, Z. Song, T. Li, X. Li, A. Y. Ogbazghi, R. Feng, Z. Dai, A. N. Marchenkov, E. H. Conrad, P. N. First, and W. A. de Heer, Science 312, 1191 (2006).

${ }^{4}$ M. I. Katsnelson, K. S. Novoselov, and A. K. Geim, Nat. Phys. 2, 620 (2006).

${ }^{5}$ H. Şahin, R. T. Senger, and S. Ciraci, J. Appl. Phys. 108, 074301 (2010).

${ }^{6}$ D. A. Dikin, S. Stankovich, E. J. Zimney, R. D. Piner, G. H. B. Dommett, G. Evmenenko, S. T. Nguyen, and R. S. Ruoff, Nature (London) 448, 457 (2007).

${ }^{7}$ D. C. Elias, R. R. Nair, T. M. G. Mohiuddin, S. V. Morozov, P. Blake, M. P. Halsall, A. C. Ferrari, D. W. Boukhvalov, M. I. Katsnelson, A. K. Geim, and K. S. Novoselov, Science 323, 610 (2009).

${ }^{8}$ J. O. Sofo, A. S. Chaudhari, and G. D. Barber, Phys. Rev. B 75, 153401 (2007).

${ }^{9}$ D. W. Boukhvalov, M. I. Katsnelson, and A. I. Lichtenstein, Phys. Rev. B 77, 035427 (2008).

${ }^{10}$ H. Şahin, C. Ataca, and S. Ciraci, Appl. Phys. Lett. 95, 222510 (2009).

${ }^{11}$ H. Şahin, C. Ataca, and S. Ciraci, Phys. Rev. B 81, 205417 (2010).

${ }^{12}$ M. Topsakal, S. Cahangirov, and S. Ciraci, Appl. Phys. Lett. 96, 091912 (2010).

${ }^{13}$ J.-C. Charlier, X. Gonze, and J.-P. Michenaud, Phys. Rev. B 47, 16162 (1993).

${ }^{14}$ Y. Takagi and K. Kusakabe, Phys. Rev. B 65, 121103 (2002).

${ }^{15}$ D. W. Boukhvalov, Physica E 43, 199 (2010).

${ }^{16}$ S.-H. Cheng, K. Zou, F. Okino, H. R. Gutierrez, A. Gupta, N. Shen, P. C. Eklund, J. O. Sofo, and J. Zhu, Phys. Rev. B 81, 205435 (2010).

${ }^{17}$ J. T. Robinson, J. S. Burgess, C. E. Junkermeier, S. C. Badescu, T. L. Reinecke, F. K. Perkins, M. K. Zalalutdniov, J. W. Baldwin, J. C. Culbertson, P. E. Sheehan, and E. S. Snow, Nano Lett. 10, 3001 (2010).

${ }^{18}$ R. R. Nair, W. Ren, R. Jalil, I. Riaz, V. G. Kravets, L. Britnell, P. Blake, F. Schedin, A. S. Mayorov, S. Yuan, M. I. Katsnelson, H.-M. Cheng, W. Strupinski, L. G. Bulusheva, A. V. Okotrub, I. V. Grigorieva, A. N. Grigorenko, K. S. Novoselov, and A. K. Geim, Small 6, 2877 (2010).
${ }^{19}$ O. Leenaerts, H. Peelaers, A. D. Hernandez-Nieves, B. Partoens, and F. M. Peeters, Phys. Rev. B 82, 195436 (2010).

${ }^{20}$ F. Withers, M. Dubois, and A. K. Savchenko, Phys. Rev. B 82, 073403 (2010).

${ }^{21}$ M. Klintenberg, S. Lebegue, M. I. Katsnelson, and O. Eriksson, Phys. Rev. B 81, 085433 (2010).

${ }^{22}$ E. Munoz, A. K. Singh, M. A. Ribas, E. S. Penev, and B. I. Yakobson, Diam. Relat. Mater. 19, 368 (2010).

${ }^{23}$ G. Kresse and J. Hafner, Phys. Rev. B 47, 558 (1993); G. Kresse and J. Furthmüller, ibid. 54, 11169 (1996).

${ }^{24}$ D. M. Ceperley and B. J. Alder, Phys. Rev. Lett. 45, 566 (1980).

${ }^{25}$ P. E. Blochl, Phys. Rev. B 50, 17953 (1994).

${ }^{26}$ M. Shishkin and G. Kresse, Phys. Rev. B 74, 035101 (2006).

${ }^{27}$ P. Giannozzi et al., J. Phys. Condens. Matter 21, 395502 (2009).

${ }^{28}$ Y. Gan, L. Sun, and F. Banhart, Small 4, 587 (2008).

${ }^{29}$ C. Ataca, E. Akturk, H. Şahin, and S. Ciraci, J. Appl. Phys. 109, 013704 (2011).

${ }^{30}$ H. Şahin, S. Cahangirov, M. Topsakal, E. Bekaroglu, E. Aktürk, R. T. Senger, and S. Ciraci, Phys. Rev. B 80, 155453 (2009).

${ }^{31}$ A. C. Ferrari, J. C. Meyer, V. Scardaci, C. Casiraghi, M. Lazzeri, F. Mauri, S. Piscanec, D. Jiang, K. S. Novoselov, S. Roth, and A. K. Geim, Phys. Rev. Lett. 97, 187401 (2006).

${ }^{32}$ S. Piscanec, M. Lazzeri, F. Mauri, A. C. Ferrari, and J. Robertson, Phys. Rev. Lett. 93, 185503 (2004).

${ }^{33}$ HSE. a hybrid functional implemented in VASP [K. Hummer, J. Harl, and G. Kresse, Phys. Rev. B 80, 115205 (2009)], is demonstrated to be as successful as the $G W_{0}$ self-energy correction method in predicting the band gaps of bulk $3 \mathrm{D}$ crystals. Consistently, for $2 \mathrm{D}$ honeycomb structures, HSE is found to yield smaller values than those of $G W_{0}$. For example, while HSE predicts the band gap of $\mathrm{CF}$ as $4.86 \mathrm{eV}, G W_{0}$ gives $7.49 \mathrm{eV}$. Similar trends are also found for $\mathrm{CH}$ and 2D boron nitride (BN). HSE and $G W_{0}$-corrected band gaps of $\mathrm{CH}(\mathrm{BN})$ are 4.51 (5.74) and $5.97(6.86) \mathrm{eV}$, respectively.

${ }^{34}$ For example, Bader [G. Henkelman, A. Arnaldsson, and H. Jonsson, Comput. Mater. Sci. 36, 254 (2006)] Löwdin [P.-O. Löwdin, J. Chem. Phys. 18, 365 (1950) ] and Mulliken [R. S. Mulliken, J. Chem. Phys. 23, 1841 (1955)] methods predict charge transfer from $\mathrm{C}$ to $\mathrm{F}$ atoms, respectively, $0.59,0.19$, and 0.06 electrons. Further analysis by calculating a planarly averaged charge density of $\mathrm{CF}$ and of free $\mathrm{C}$ and $\mathrm{F}$ atoms, where the charge due to the tails of orbitals are carefully accounted, deduces that the total of 0.11 electrons are transferred from buckled graphene to each $\mathrm{F}$ atom. 